\title{
Providing research services to your campus without a library
}

$\mathbf{P}$

enn State-Berks is one of 24 commonwealth campuses in the Pennsylvania State University (PSU) system. With around 2,800 students, it is a small, tight knit community, yet remains one of the largest campuses outside of University Park.

While the campus is spacious and beautiful, one feature it lacks is an adequate student center, which makes the Thun Library the heart of the campus. Not only does the library provide traditional and valued services, such as access to textbooks on reserve, computer and software access, and research assistance, it is also a location where students gather to study, hang out between classes, and conduct group work. With a large commuter population, the library offers vital spaces for those without dorm rooms to complete their work. In addition, several other departments are also housed within the building, including the Center for Teaching and Learning along with the information technology (IT) help desk.

\section{Unexpected emergency closure}

In October 2017, the week before midterms, mold was detected within the building. As it was pervasive and rapidly spreading, the library had to close for emergency maintenance to rectify the issue. With the sudden closure and no clear timeline in place, our staff had to scramble to brainstorm and implement solutions. Since library services are essential to student success, a complete cessation of services was out of the question. But how could we, specifically as reference librarians, soldier on without access to a dedicated area to supply services like research assistance and library instruction? Space is a precious and rare commodity on the Berks campus, and there was none left to spare for librarians to set up camp. With other staff requiring relocation, even conference rooms and lobbies filled up with services, such as the IT help desk, course reserve checkouts, interlibrary loan functions, and general staff workspace. Without the library, buildings on campus overflowed with students. While walking around, it was not unusual to see every open lounge chair filled within each building's hallways or to witness individuals tucked into remote corners sitting on the floor. Even one of the authors had to participate in a webinar behind a vending machine. To put it simply, there was no room at the inn for us.

Library instruction sessions proved a straightforward fix. Our administrative assistant worked to piece together available computer classrooms that could accommodate our existing class schedules. While there were a few hiccups along the way, including a double booking incident and a few rescheduled sessions, for the most part all classes could continue as usual throughout campus.

Alexandria Chisholm is reference and instruction librarian, email: aec67@psu.edu, Brett Spencer is reference and instruction librarian at Penn State-Berks, email: dbs21@psu.edu

(c) 2019 Alexandria Chisholm and Brett Spencer 
Solutions to providing research services were a bit more complicated. Here at Penn State-Berks we have an established and highly valued drop-in and appointment-based research consultation service that we knew would be missed. We were concerned with the negative impact to students on campus if we could not locate an area to establish ourselves during the building closure. Our initial solution had been to direct students through signage on campus and our social media accounts to use the PSU Libraries' Ask A Librarian chat service, which is staffed by ourselves and librarians at the other PSU campuses. However, we knew this would not replace the personalized in-person research assistance to which they had grown accustomed.

\section{The Writing Center to the Penn State-Berks Writing Center. rescue}

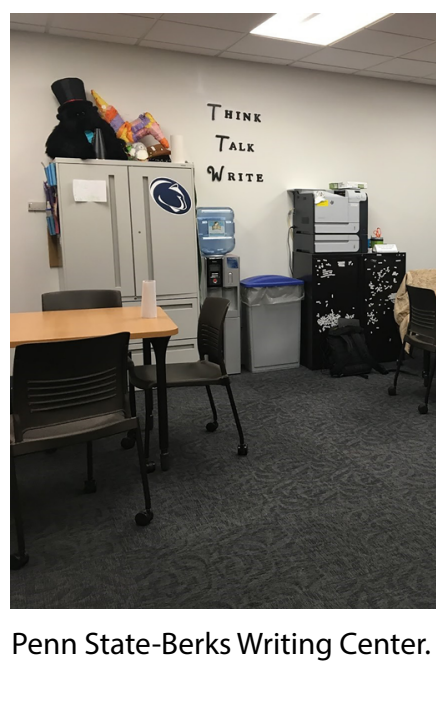

While library staff found hallway space to offer access to course reserves, the librarians were lucky enough to discover a fruitful partnership through one of our student workers, who also worked as the head writing tutor in the campus' Writing Center (WC). Having insider knowledge of both departments, she knew that librarians needed a space to assist students and that the WC hoped to extend its hours. From this unique perspective, she was able to suggest a collaboration and connect us with the WC.

The interim Writing Center Director, Jayne Brown, graciously offered us space to provide research services within the WC. We had access to computers and printers, but most importantly space on campus to make ourselves available to our student community. By accommodating librarians' need for a workspace, the WC also increased student use of their space and services, providing justification for a long-held goal of extending its hours to include Fridays. This mutually beneficial scenario proved to be helpful in the short and long term for both parties, and seemed like an effortless collaboration, given the related nature of our work assisting students in their research and writing.

To promote the new setup, we sent out a campus-wide email to faculty, staff, and students advertising that we would be in the Writing Center from 10 a.m. to 3 p.m. Monday

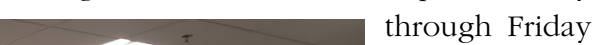
with additional times by appointment.

$\mathrm{H} \mathrm{o} \mathrm{w} \mathrm{e} \mathrm{v} \mathrm{-}$ er, attempting to communicate our whereabouts and availability placed an added burden on the busy writing tutors, and we quickly acted to alleviate some of that responsibility by providing a simple tool to place the communication back into the hands of students seeking librarian assistance. Within a week after launching services in the WC, we created cards with our temporary hours, names, and email addresses so that tutors could give them to students.

\section{From contingency plan to revelation}

During our six weeks of displacement in the WC, we learned a multitude of beneficial lessons that any librarian can profit from. The biggest moral highlighted throughout our experience was that writing and research are inextricably connected academic skills that have blurred contexts. Therefore, both librarians and tutors encounter students reaching out for intersecting assistance. While we can encourage students to follow 
up with the appropriate contact who is most equipped to assist them, anecdotally we have found the added step hinders successful follow through.

During our stay, librarians had the exciting and unique experience of "tag teaming” appointments with the writing tutors. In many cases, this teamwork benefited students immensely. Since writing and research are so interconnected, it was enormously helpful for librarians to aid students on how to develop their topics, find appropriate sources, evaluate sources that had questionable validity, and cite their sources accurately, while tutors handled developing arguments, paraphrasing/ quoting, along with the mechanics and style of writing.

Being colocated enabled these collaborations to occur naturally and without much formal setup, a scenario that will likely never again happen on such a casual basis. Nonetheless, it opened our eyes to the potential impact of intentional partnerships between academic libraries and writing centers.

As librarians, we also developed a clear understanding of how the WC's services were scheduled and functioned. When we returned to our routine schedules, we could accurately refer students using experiential knowledge. Writing tutors often deal with inflated expectations from students. Now we could help prime referrals so they knew the type of assistance they could expect from writing tutors and how to best prepare for their appointments to get the most out of their sessions. In turn, the experience also illuminated to writing tutors how librarians can assist students with their research. Many tutors were happy to discover that there were resources on campus that handled some of the skills with which they were less comfortable, such as source evaluation and selection, as well as citation.

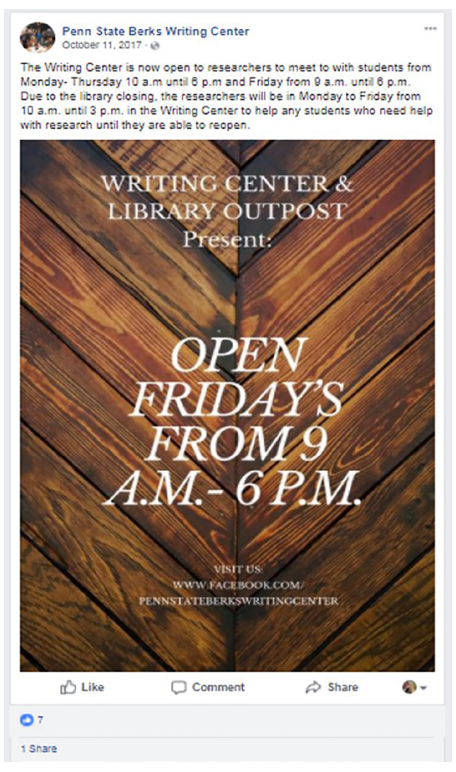

The referral system between our two departments is stronger than ever, with no misconceptions about how services work, the limitations of our assistance, or the intersecting support that can be obtained from librarians and writing tutors. This is a huge win for our student population.

Most of these lessons were things we conceptually envisioned and wanted to embrace. In fact, prior to the emergency closure, we had already begun planning a Research and Writing Carnival, which would bring together

Facebook post from Penn Statewriting tutor and librarian assistance. This event serendipitously ended up occurring a mere two weeks after 
we returned home to our library building. Our extended stay in the WC made this event far more authentic, as we had already established relationships with tutors, practiced tag-teaming consultations, and gained a more thorough understanding of our intersecting roles.

Creating a relationship with your campus writing center, along with its director and tutors, is critical to developing a holistic approach to student research and writing services. Our Research and Writing Carnival is just one way that we plan to continue cultivating this vital relationship. We also have plans to set up formal training workshops specifically for tutors to help them develop strategies to assist students with intersecting skills, like citation and ethical use of information. Overall, we are simply excited to see the many creative ways we continue to build this partnership to improve services to students at Penn StateBerks-and the emergency closure had a silver lining by fostering these collaborations.

\section{Not all who wander are lost}

Another unexpected benefit to being professionally adrift sprang

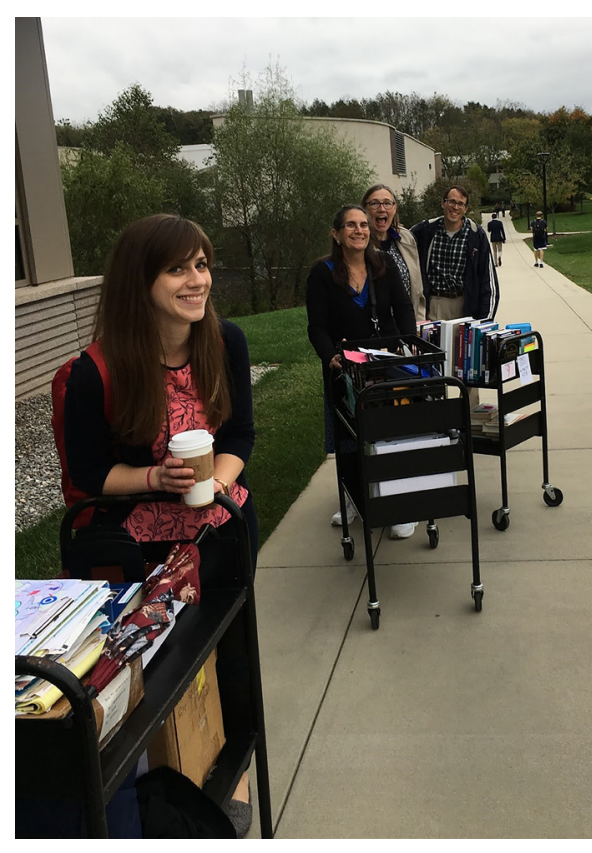
Library staff transport course reserves and other materials from the Thun Library to another location on campus. from how it forced us out of the library building and deep into the campus community. Nothing brings people together like shared suffering. Throughout our time vacated from the library, faculty, staff, and students all over campus expressed sympathy and words of encouragement. Students would stop us to discuss updates and solutions to issues that were arising in the absence of the library building. We interacted with faculty and staff with whom we typically do not have the opportunity to collaborate.
Overall, our displacement made the seemingly invisible value of our resources and services exceedingly evident. The sight of library staff camped out under the stairs of an academic building with a mobile course reserve and circulation station, or librarians trudging about the campus with loaded backpacks, highlighted the dedication and value of what we bring to our academic community.

\section{Takeaways}

What if your library falls prey to a mold monster or some other unfortunate event, but your campus continues to function as usual? Here are some things we learned that we hope will help you make the best of a bad situation:

- Communication is key. Immediately assure the campus by email that you will create backup research services and quickly settle on a plan to implement.

- Remind campus faculty, staff, and students that they can access your virtual references services. Reinforce this service with extra staffing.

- Make the best of the situation by practicing embedded librarianship. Would a writing center be an appropriate place to relocate? Could librarians find spaces in their liaison departments? Are there high traffic spaces on campus where you could set up shop? Figure out what works best for your campus setting.

- Schedule alternate computer classrooms so you can continue offering instruction. If computer classrooms are unavailable, ask faculty to encourage their students to

(continues on page 99) 
library working on a single project, they are focused on several. These students are well-balanced and well organized, and have come to expect the stability that the library provides for their hectic schedule. They are driven by goals and achievement, and need a space where the services are reliable and the tools they need are in supply. These are just a few examples of the core patrons best served by the late-night scene, but there are dozens of categories.

Understanding all these things and trying to remain mindful of personalities drawn to this type of environment is a rather large part of what do as overnight information associates. It's one of those duties that's difficult to put into context, and even harder to plan accommodations for. It's a bit of a magical quality that's simply evolved during our service as the sole representatives of the library during this time slot. We're part sympathetic bartender, part father-figure, part gate-keeper, part medic, part library professional. We know when silence is golden, and when a listening ear is needed. We are observant of those who are weary and in need of rest versus those in distress and in need medical assistance. We can discern the difference between the whinings of a procrastinator and the pleadings of a genuinely frustrated, overworked student in need of a miracle. We strive each night to master the art of blending policy enforcement with a shared humanity that keeps us engaged and motivated about serving and advocating for the late-night patrons of the Georgia Tech Library.

\section{Conclusion}

As we move toward implementing LibraryNext, an ambitious reimagining of the Georgia Tech Library, we know that we have to mold overnight use and services to what is best for the students. Before we even started the renewal process, we knew it would be different at night, and instantly began thinking of ways to improve specifically the overnight experience. It is because we know through our time here that there are differences, that a one-size-fits-all approach is not the best strategy for users with diverse needs and that we are always looking towards providing an experience as necessary, unique, and enjoyable as possible at night. $\boldsymbol{2}$

("The displaced librarian," continues from page 95)

bring laptops to their regular classrooms. You might even be able to borrow laptops from your campus technology department. Post your handouts online, since printing and copying will be tricky during a displacement.

- Prepare for a nomadic librarian lifestyle. Procure laptops and backpacks that will permit you to rove around campus outside your temporary reference area. It can be difficult to foresee where students will congregate after a library closure. Leave yourself the flexibility to go directly to the students in addition to maintaining a relocated reference desk.

- Maintain a positive attitude. Every day is filled with new and different challenges when you are removed from your typical environment. Go with the flow and embrace the uncertainty with grace.

An emergency closure is definitely an unwelcome disruption to professional life. However, students will still need research support in order for learning to occur. Strategically seize the opportunity to do campus outreach and connect with faculty, staff, and students whether they are library novices or die-hard supporters. Being a displaced librarian opens up doors to strengthening campus partnerships and making embedded librarianship not just a reality but a necessity. The experience has certainly taught us that while the physical library is essential to the campus, the work we do as librarians transcends the four walls of our building. $\pi$ 